\title{
Efectos del alta hospitalaria temprana en el periodo posparto en la Clínica del Prado, Medellín, Colombia*
}

\section{Effects of early discharge in the postpartum period in la Clínica del Prado, Medellín, Colombia}

\section{Efeitos da alta hospitalar precoce no período pós-parto na Clínica del Prado, Medellín, Colômbia}

Fecha de recepción: 29-01-13 Fecha de aceptación: 04-03-14

doi:10.11144/Javeriana.RGYPS13-26.eaht

\author{
Arturo Cardona-Ospina** \\ Jaime Ordoñez-Molina*** \\ Elsa María Vásquez-Trespalacios**** \\ Laureano Mestra-Palomino***** \\ Raúl Alejandro García-Posada******
}

Artículo de investigación. Proyecto de investigación: Efectos del alta hospitalaria temprana en el periodo postparto en la Clínica del Prado, Medellín, Colombia. Inicio: septiembre de 2009; finalización: junio de 2012. Clínica del PradoUniversidad CEs. Correo electrónico: acardona@clinicadelprado.com.co

** Médico y cirujano, Universidad Libre de Cali. Fetólogo, Fetus, Brasil. Coordinador académico, Clínica del Prado, Medellín, Colombia.

*** Médico y cirujano, Universidad de Antioquia Ph.D. en Epidemiología. Coordinador del Centro de Evaluación de Tecnologías en Salud (Cetes), Universidad CES.

***** Bióloga. Magíster en Epidemiología, Universidad de Antioquia. Coordinadora del Centro de Investigaciones de la Clínica del Prado. Docente de la Universidad CES.

******* Médico, Escuela de Medicina Juan N. Corpas. Magíster en Epidemiología, Universidad Ces. Correo electrónico: laureanomestra@gmail.com

*t**ese Médico y cirujano, Universidad Pontificia Bolivariana. Especialista en Ginecología y Obstetricia, Universidad de Antioquia. Especialista en Medicina Materno Fetal, Hospital Clinic de Barcelona. Correo electrónico: alegapo@hotmail.com 


\section{Resumen}

Problema: evaluar el efecto del alta hospitalaria temprana en pacientes sanas. Objetivo: establecer el riesgo de complicaciones en pacientes obstétricas con alta temprana respecto a aquellas que egresan después de 24 horas. Métodos: cohorte prospectiva con seguimiento postparto. Resultados: 750 pacientes, edad promedio de 24,5 años y edad gestacional de 38,5 semanas. El porcentaje de consulta luego del alta fue 2,0\%. El efecto del alta hospitalaria temprana sobre la necesidad de consultar luego del egreso tuvo un $\mathrm{RR}=0,62$ (IC 95\%=0,22-1,73). Estratificando según vía del parto se encontró en las pacientes con parto vaginal un RR $=0,11$ (IC $95 \%=0,014-0,933)$, y en aquellas sometidas a cesárea un $\mathrm{RR}=1,91$ (IC $95 \%=0,247-13,32$ ). Conclusión: no se presentaron más complicaciones en el grupo con egreso antes de las 24 horas. El alta hospitalaria temprana se comportó como factor protector sobre la necesidad de consultar a una institución hospitalaria en las pacientes con parto vaginal.

Palabras clave: alta del paciente; complicaciones posoperatorias; parto obstétrico; cesárea; periodo de posparto; cohorte

Palabras clave descriptor: alta del paciente; complicaciones postoperatorias; parto obstétrico; cesárea; periodo de postparto; estudios de cohortes

\section{Abstract}

Problem: Assess early discharge in healthy patients Aim: To establish the risk of obstetric complications in patients with early discharge compared to those who leave after 24 hours. Methods: prospective cohort with postpartum follow up. Results: 750 patients, mean age of 24,5 years and average of gestational age of 38,5 weeks. The overall rate of consulting to an ER after discharge was $2,0 \%$. The effect of early discharge on the need to consult had a RR $=0,62(95 \%$ CI: $0,22-1,73)$. By adjusting the analysis by the way of delivery, in patients whose delivery was vaginal, the effect had a RR $=0,11(95 \% \mathrm{CI}: 0,014-0,933)$ and in patients undergoing caesarean section, the effect had a RR $=1,91(95 \% \mathrm{CI}: 0,274-13,32)$. Conclusion: there was a protective effect of early discharge on the need to consult a hospital, in patients who delivered vaginally. Keywords: patient discharge; postoperative complications; obstetric delivery; cesarean section; postpartum period; cohort

Keywords plus: patient discharge; postoperative complications; delivery obstetric; cesarean section; postpartum period; cohort study

\section{Resumo}

Problema: avaliar o efeito da alta hospitalar precoce em pacientes sanas. Objetivo: determinar o risco de complicações em paciente obstétrica com alta precoce, no que diz respeito daquelas que afastam após 24 horas. Métodos: coorte prospectiva com . Resultados: 750 pacientes, idade média de 24,5 anos e idade gestacional de 38,5 semanas. A porcentagem de consulta após a alta foi $2,0 \%$. O efeito da alta hospitalar precoce sobre a necessidade de consultar após o egresso obteve um RR $=0,62$ (IC $95 \%=0,22-1,73$ ). Estratificando de acordo com a via do parto foi encontrado em pacientes com parto natural um RR $=0,11$ (IC 95\%=0,014-0,933), e em aquelas submetidas a cesariana, um RR $=1,91$ (IC $95 \%=0,247$ - 13,32). Conclusão: não apresentaram-se mais complicações na turma com egresso antes das 24 horas. A alta hospitalar precoce se comportou como fator protetor sobre a necessidade de consultar uma instituição hospitalar nas pacientes com parto natural.

Palavras chave: alta de paciente; complicações pós-operatórias; parto obstétrico; cesariana, período de pós-parto, coorte

Palavras chave descritores: alta do paciente; complicações pós-operátorias; parto obstétrico; cesárea; período pós-parto; estudos de coortes 


\section{Introducción}

La principal razón para la hospitalización durante el periodo posparto es identificar las complicaciones maternas y neonatales posparto (1), pero el tiempo que debe esperarse antes del alta hospitalaria es un tema que ha sido de particular importancia en la atención en salud.

Desde el decenio de 1950 se ha observado una disminución gradual en el tiempo que las madres permanecen en el hospital luego del parto (2), pasando de un periodo de hospitalización de entre 8 y 14 días en pacientes con un parto vaginal sin complicaciones, a periodos de dos a tres días, o incluso menores. En algunas partes de Estados Unidos, la estancia hospitalaria es de 12 a 24 horas en pacientes con parto vaginal sin complicaciones, y de 48 a 72 horas en aquellas que tuvieron una cesárea sin complicaciones (2).

En las pacientes que tuvieron parto vaginal, tradicionalmente se ha definido el alta temprana como el alta hospitalaria en un tiempo menor a las 48 horas posparto (2), pero en la práctica, las definiciones de lo que constituye el alta temprana varían entre los diferentes países (2). Esta variación se refleja en las publicaciones sobre alta temprana en las que las participantes que recibieron fueron dadas de alta a las 12 horas posparto, y tan tarde como tres a cuatro días luego del parto fueron consideradas en la misma categoría de alta temprana (2).

A pesar de que tradicionalmente se ha contado con evidencia contradictoria, en la actualidad existen estudios que soportan el alta temprana como una medida segura, tanto para la madre como para el bebé $(3,4)$.

La política institucional de alta en la Clínica del Prado es dar el alta a las pacientes cuyo parto fue por vía vaginal desde las 8 horas y en lo posible no más de 24 horas después del parto; a las pacientes con cesárea, el alta es desde las 12 y hasta las 24 o 36 horas poste- riores al parto. Esta política solo es aplicable para pacientes sin riesgos adicionales y sin enfermedades adicionales.

El objetivo de esta investigación es determinar el riesgo del alta temprana sobre la necesidad de consulta de urgencias en los primeros cinco días durante el periodo posparto, en pacientes que tuvieron un parto vaginal o una cesárea sin complicaciones.

\section{Materiales y métodos}

Con previa aprobación del Comité de Ética de la Clínica del Prado en la ciudad de Medellín, Colombia, se realizó un estudio de cohorte prospectivo, en el que la población estuvo constituida por madres sanas con parto vaginal y cesárea atendidas en la institución entre julio y octubre de 2009.

La cohorte expuesta estuvo comprendida por las pacientes que no presentaron ninguna complicación durante el parto y sin patologías en el embarazo, quienes egresaron de la institución en las primeras 24 horas después del parto o cesárea. La cohorte no expuesta estuvo comprendida por las pacientes que no presentaron ninguna complicación durante el parto y sin patologías en el embarazo, quienes egresaron de la institución después de 24 horas del parto o cesárea. La asignación de las pacientes a los grupos se realizó de acuerdo con la duración de los trámites administrativos para el egreso de las pacientes. En el caso de aquellas cuyo trámite tuviera una duración mayor, se les solicitó permanecer en la institución más de las 24 horas establecidas como punto de corte. No se tuvo la negativa de ninguna madre en permanecer más tiempo del establecido en la clínica después del parto.

Se realizó un muestreo no probabilístico, incluyendo la totalidad de la población al estudio y seleccionando a las pacientes que cumplieran 
con los criterios de inclusión y no cumplieran ningún criterio de exclusión. Ingresando a la investigación un total de 750 pacientes.

Se incluyeron las pacientes que habían tenido partos vaginales o instrumentados con evolución normal y aquellas que habían sido sometidas a una cesárea electiva o urgente con evolución normal. Se excluyeron las pacientes con preeclampsia moderada o severa, hipertensión arterial crónica, hemorragia posparto, embarazo múltiple, taquicardia materna posparto, intolerancia a la vía oral, infección intraamniótica, infección bacteriana en tratamiento, desgarro perineal grado IV, diabetes gestacional y diabetes mellitus tipos I o II, y aquellas cuyos neonatos debieron quedarse en observación.

Las pacientes incluidas se siguieron telefónicamente durante cinco días, con el fin de identificar si ellas o los bebés debieron consultar a alguna institución de salud por la presencia de alguna complicación médica durante este periodo. Solo se tuvieron en cuenta las consultas presenciales, en las que la madre acudiera a las instalaciones de la Clínica del Prado. Se hicieron dos llamadas: a las 48 horas del egreso y a los 5 días del mismo por parte de personal de la Clínica, que se estandarizó previamente, y que utilizó una lista de chequeo para describir los motivos de consulta de las pacientes o de los bebés. Esta información se contrastó con los registros de ingreso por consulta de cada una de las participantes.

Se estableció la magnitud del efecto de la exposición a través del cálculo del riesgo relativo así como de los intervalos de confianza del $95 \%$.

\section{Resultados}

El total de pacientes expuestas fue de 388 y el de no expuestas 362. El promedio de edad y su desviación estándar en el grupo de pacientes expuestas fueron 25,7 años ( \pm $6,47)$, y el de las pacientes no expuestas 26,1 años $( \pm 6,47)$. Asimismo, el promedio de edad gestacional y su desviación estándar en el grupo de pacientes expuestas fueron 38,6 semanas $( \pm 1,35)$, y el de las pacientes no expuestas 38,6 semanas $( \pm 1,52)$. No se observaron diferencias estadísticamente significativas en la edad de la madre ni en la edad gestacional entre la cohorte expuesta y la no expuesta $(\mathrm{p}=0,64$ y $\mathrm{p}=0,65$ respectivamente) (tabla 1$)$

TABLA 1. Características de las pacientes evaluadas

\begin{tabular}{|c|c|c|c|c|c|}
\hline \multirow{3}{*}{ Tiempo del alta } & \multicolumn{4}{|c|}{ Tiempo del alta } & \multirow{3}{*}{$\begin{array}{l}\text { Valor } \\
\text { de } P\end{array}$} \\
\hline & \multicolumn{2}{|c|}{ Menor o igual a $24 \mathrm{~h}(n=388)$} & \multicolumn{2}{|c|}{ Mayor a 24h $(n=362)$} & \\
\hline & Promedio & $\begin{array}{c}\text { Desviación } \\
\text { estándar }\end{array}$ & Promedio & $\begin{array}{c}\text { Desviación } \\
\text { estándar }\end{array}$ & \\
\hline Edad de la madre & 25,73 & 6,47 & 26,09 & 6,47 & 0,64 \\
\hline Edad gestacional & 38,6 & 1,35 & 38,55 & 1,52 & 0,65 \\
\hline Tiempo parto egreso & 17,61 & 4,07 & 32,75 & 8,36 & 0,000 \\
\hline Embarazos previos & 1,9 & 1,12 & 1,81 & 0,99 & 0,250 \\
\hline Partos previos & 1,36 & 1,06 & 1 & 1 & 0,000 \\
\hline Abortos previos & 0,2 & 0,52 & 0,18 & 0,47 & 0,61 \\
\hline Cesáreas previas & 0,3 & 0,6 & 0,6 & 0,8 & 0,000 \\
\hline Ectópicos previos & 0,02 & 0,15 & 0 & 0,07 & 0,04 \\
\hline
\end{tabular}

Fuente: elaboración propia 
Existen diferencias estadísticamente significativas con respecto al número de partos, cesáreas y embarazos ectópicos previos entre la cohorte expuesta y la no expuesta $(\mathrm{p}<0,05)$ (tabla 1$)$.

Con respecto al tiempo de hospitalización de la paciente entre el momento del parto y su egreso, el promedio de tiempo de hospitalización posparto y su desviación estándar en el grupo de pacientes expuestas fueron 17,6 horas $( \pm 4,07)$, y el de las pacientes no expuestas 32,75 horas $( \pm 8,36)$ (tabla 1$)$.

Con referencia a la vía de terminación del embarazo, la proporción de cesáreas en el grupo de pacientes expuestas (hospitalización menor de 24 horas) fue de $20,6 \%$ y en el grupo de pacientes no expuestas esta proporción alcanzó el 42,3\%. Estas diferencias son estadísticamente significativas (tabla 2).

A la totalidad de la población se le realizaron dos llamadas telefónicas, la primera 48 horas después del egreso hospitalario y la segunda 5 días después de dicho egreso. Se localizó a todas las pacientes para identificar si durante ese tiempo debieron buscar atención médica. En el grupo de expuestas se identificaron tres pa- cientes que habían solicitado atención médica durante este periodo, en tanto que en el grupo de no expuestas se identificaron 8 pacientes.

$\mathrm{Al}$ determinar el efecto del alta temprana sobre la necesidad de consultar en los primeros cinco días luego del alta hospitalaria, se obtuvo un $\mathrm{RR}=0,76$ (IC 95\% 0,41 - 1,43). De ahí se concluye que un alta temprana no se relaciona con una mayor necesidad de consultar a una institución de salud en comparación con las pacientes que recibieron un alta tardía.

$\mathrm{Al}$ estratificar este análisis según la vía del parto, en aquellas pacientes que tuvieron un parto por vía vaginal se obtuvo un $\mathrm{RR}=0,11$ (IC 95\% = 0,014 - 0,933). En estas pacientes un alta temprana se comportó como un factor protector ante la necesidad de consultar a la institución de salud, en comparación con las pacientes que recibieron el alta luego de las 24 horas (tabla 3 ).

En las pacientes sometidas a cesárea se obtuvo un $\mathrm{RR}=1,91(\mathrm{IC} 95 \%=0,274-13,32)$. Con base en este dato se llegó a la conclusión de que no existen diferencias estadísticamente significativas en la necesidad de consultar

TABLA 2. Distribución del tipo de PaRto SEgún el MOMento del alta en las gestantes.

\begin{tabular}{|c|c|c|c|c|c|c|}
\hline \multirow{2}{*}{$\begin{array}{c}\text { Tipo de } \\
\text { parto }\end{array}$} & \multicolumn{2}{|c|}{ Menos de 24 horas } & \multicolumn{2}{|c|}{ Más de 24 horas } & \multirow[t]{2}{*}{ Total } & \multirow[t]{2}{*}{ Valor de $p$} \\
\hline & $\mathbf{n}$ & $\%$ & $\mathbf{n}$ & $\%$ & & \\
\hline Cesárea & 80 & 20,62 & 153 & 42,27 & 233 & \\
\hline Vaginal & 308 & 79,38 & 209 & 57,73 & 517 & 0,000 \\
\hline Total & 388 & 100 & 362 & 100 & 750 & \\
\hline
\end{tabular}

Fuente: elaboración propia

TABLA 3. Distribución de las PaCientes Que TUVIERON NECESIDAd de VisitaR UNa INSTITUCión de SALUd LUEGO DEL ALTA LUEGO DE PARTO VAGINAL

\begin{tabular}{|l|c|c|c|c|}
\hline \multirow{2}{*}{ Tipo de alta } & \multicolumn{3}{|c|}{ Necesidad de visitar institución de salud } \\
\cline { 2 - 4 } & $\mathrm{N}$ & $\%$ & Riesgo Relativo & IC 95\% \\
\cline { 1 - 3 } Menor o igual a 24 horas $(\mathrm{n}=308)$ & 1 & 0,30 & 0,11 & $(0,014-0,933)$ \\
\hline Mayor de 24 horas $(\mathrm{n}=209)$ & 6 & 2,90 & & \\
\hline
\end{tabular}




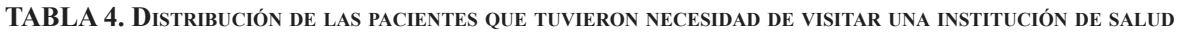
LUEGo DEL ALTA LUEGo DE CESÁREA

\begin{tabular}{|l|c|c|c|c|}
\hline \multirow{2}{*}{ Tipo de alta } & \multicolumn{4}{|c|}{ Necesidad de visitar institución de salud } \\
\cline { 2 - 5 } & $\mathrm{N}$ & $\%$ & Riesgo Relativo & IC 95\% \\
\cline { 1 - 4 } Menor o igual a 24 horas $(\mathrm{n}=80)$ & 2 & 2,50 & \multirow{2}{*}{1,91} & $(0,274-13,32)$ \\
\hline Mayor de 24 horas $(\mathrm{n}=153)$ & 2 & 1,30 & & \\
\hline
\end{tabular}

Fuente: elaboración propia

por motivos de salud entre las pacientes con alta temprana y aquellas con alta posterior a las 24 horas, luego de cesárea (tabla 4).

\section{Discusión}

El hallazgo principal de este estudio es la ausencia de diferencias en la necesidad de consultar por motivos de salud luego del parto o cesárea. Esto resulta similar a lo reportado por otras investigaciones (5-11).

En cuanto a las hospitalizaciones neonatales, solo se presentó una durante el periodo de observación y fue en el grupo de pacientes que estuvieron hospitalizadas más de 24 horas. Estos hallazgos son similares a los reportados por otros investigadores $(5,6,8,9,12)$, pero debe aclararse que en dichos estudios el tiempo de seguimiento de los neonatos fue de aproximadamente ocho semanas, y en esta investigación solo fue de cinco días, ya que en Colombia los bebés y sus madres siempre son evaluados de forma ambulatoria una semana después del parto.

La falta de asociación que se observó en esta investigación entre el alta temprana de la madre y el bebé y sus resultados clínicos, es similar a la conclusión planteada por Brown et ál. (4) en un metanálisis publicado en 2010. Incluso, en el presente estudio se observó que en el subgrupo de pacientes cuyo parto fue vaginal, el alta temprana tenía un efecto protector sobre los resultados clínicos maternos y neonatales, consistente con lo observado en este estudio.

Lo anterior podría explicarse en parte porque el grupo de pacientes que fueron dadas de alta en las primeras 24 horas posparto estuvo compuesto mayoritariamente $(79,4 \%)$ por pacientes cuyo parto fue vaginal, a diferencia del grupo cuyo egreso fue después de las 24 horas posparto, en el que la proporción de pacientes con parto vaginal fue de $57,7 \%$. Lo anterior podría interpretarse no solo como que el alta temprana en las pacientes de bajo riesgo no tiene asociación con resultados negativos en las madres y los neonatos, sino que además esta tendría un efecto protector sobre aquellas cuya vía del parto fue vaginal.

Asimismo, es importante mencionar que no hubo rehospitalizaciones de las madres o de los neonatos durante la primera semana posparto en ninguno de los dos grupos de observación. En un metanálisis hecho por Brown et ál. (2), al analizar las rehospitalizaciones neonatales que tuvieron lugar dentro de las tres a ocho semanas posparto, no se encontraron asociaciones con el alta hospitalaria temprana $(\mathrm{RR}=1,23$ [IC 95\% $=$ 0,37 - 4,04]). De igual forma, en el mismo estudio tampoco se encontraron asociaciones con las rehospitalizaciones maternas $(\mathrm{RR}=0,76[\mathrm{IC} 95 \%=0,17-3,37])$.

El presente estudio, en virtud de su carácter observacional, tiene mayor posibilidad de presentar sesgos de selección que los estudios experimentales. De hecho, la vía del parto 
en la mayor parte de las pacientes expuestas fue vaginal y en el caso de las no expuestas fue abdominal, lo cual se explica porque es esperado que las pacientes con parto por vía vaginal se recuperen más rápido que aquellas cuya vía del parto fue por cesárea. A pesar de esto, los autores consideramos que esta situación no afecta las conclusiones principales, pues lo que se pretende evaluar es la efectividad de una política de atención por medio de la observación de su aplicación. Además, desde el punto de vista ético, sería difícil hacer un estudio experimental para evaluar esta política de atención, pues una paciente y su familia no entenderían por qué no pueden irse para su casa lo más pronto posible con su bebé si se encuentran en buenas condiciones clínicas. Sin embargo, en este estudio no se tuvo la negativa de ninguna madre para egresar de la institución según la cohorte de estudio a la cual pertenecía.

Teóricamente, desde el punto de vista económico y de seguridad del paciente, el alta hospitalaria temprana ofrece varias ventajas. La primera es la disminución del riesgo de infecciones intrahospitalarias. Por definición, una infección hospitalaria o nosocomial es un proceso contraído en un centro hospitalario. La infección adquirida en el medio hospitalario es aquella que se manifiesta durante o después de 48 horas de la internación y que no estaba presente ni incubándose en el momento del ingreso (13). Lo anterior significa que entre más tiempo pase hospitalizada una paciente, tendrá mayor riesgo de desarrollar una infección intrahospitalaria. De hecho, el indicador con base en el cual se reportan estas, solo considera como población de riesgo aquellos pacientes que estén hospitalizados más de 48 horas (14).

Una de las ventajas más importante del alta hospitalaria temprana residiría en la disminución del riesgo de desarrollar este tipo de infecciones, lo cual afectaría la morbilidad, mortalidad y calidad de vida de las pacientes.
Esta situación también se relaciona con aspectos económicos, ya que no habría que dedicar recursos económicos al tratamiento de patologías que potencialmente eran prevenibles. Datos de los hospitales públicos de Victoria (Australia) muestran que cada día de estancia hospitalaria acarrea un riesgo de infección del $17,6 \%$ y cada noche adicional aumenta el riesgo de infección en un 1,6\% (15).

Ramirez-Villalobos et ál. evaluaron 303 mujeres con parto vaginal atendidas en un hospital público de la Ciudad de México, sin complicaciones antes del egreso hospitalario categorizado como temprano si tenía lugar antes o a las 24 horas luego del parto y reportan que las mujeres con alta temprana posparto con adecuado control prenatal tuvieron menos síntomas de complicaciones en el puerperio inmediato (RM: 0.36; IC $95 \%=0,17-0,76)$, lo cual es consistente con lo encontrado en este estudio (16).

De igual forma, Ford et ál., al evaluar el cambio en las readmisiones maternas de acuerdo con la longitud de la estancia hospitalaria en New South Wales entre 2001 y 2007, concluyen que a pesar de la disminución en la duración de la estancia hospitalaria posparto, no hubo incremento, sino una disminución en la tasa de readmisiones hospitalarias (17).

Asimismo, el alta hospitalaria temprana permitiría que los hospitales pudiesen ser más eficientes en la utilización de su infraestructura, aumentando el indicador de giro cama, entendido este último como la rotación media de las camas y expresa cuántos pacientes pasan en un periodo dado, en promedio por cama disponible.

Desde el punto de vista de los costos asociados a la atención en salud, Petrou et ál. compararon a través de un ensayo aleatorizado controlado con análisis de costos, la relación costo-efectividad de un alta temprana con cuidado en casa vs. la estancia hospitalaria 
tradicional, y encontraron que los resultados clínicos y psicosociales fueron similares en los dos brazos de observación, en concordancia con lo encontrando en este estudio, y el alta temprana produjo una disminución significativa de los costos hospitalarios posnatales (18). De modo similar, Sainz-Bueno et ál. (19) reportan no haber encontrado diferencias significativas en las tasas de rehospitalización materna ni en neonatos, cuando se compara un alta temprana con seguimiento domiciliario con la estancia hospitalaria tradicional, y a nivel de costos observaron una disminución de entre 18 y $20 \%$ con el alta temprana de las pacientes, reportando también una satisfacción superior en las pacientes con alta temprana (19).

Los autores concluyen que el alta hospitalaria temprana en las primeras 24 horas posparto, en pacientes que no tengan ninguna contraindicación para egresar del hospital, no tiene ninguna asociación con reingresos hospitalarios del neonato o de su madre. Además, puede disminuir el riesgo de exposición a infecciones intrahospitalarias, al permanecer las pacientes hospitalizadas menos de 48 horas.

\section{Referencias bibliográficas}

1. Riley L, Lemons JA, Lockwood C, Blackmon L, editores. Guidelines for perinatal care. Sexta edición. Washington D.C.: American Academy of Pediatrics, The American College of Obstetrician and Gynecologists; 2007.

2. Brown S, Small R, Argus B, Davis PG, Krastev A. Early postnatal discharge from hospital for healthy mothers and term infants. Cochrane Database of Systematic Reviews. The Cochrane Library. 2010; 10. doi: 10.1002/14651858.CD002958.pub3

3. Madden JM, Suomerai SB, Lieu TA, Mandl KD, Zhang F, Ross-Degnan D. Effects of a law against early postpartum discharge on newborn follow-up, adverse events, and HMO expenditures. N Engl J Med. 2002; 347: 2031-8.

4. Mandl KD, Homer CJ, Harary O, Finkelstein JA. Effect of a reduced postpartum length of stay program on primary care services use by mothers and infants. Pediatrics 2000; 106 (4): 937-41.

5. Hellman LM, Kohl SG. Early hospital discharge in obstetrics. Lancet. 1962; 1 (7223): 227-32.

6. Waldenström U, Sundelin C, Lindmar G. Early and late discharge after hospital birth. Health of mother and infant in the postpartum period. Ups J Med Sci. 1987; 92: 301-14.

7. Carty E, Bradley C. A randomized, controlled evaluation of early postpartum hospital discharge. Birth 1990; 17 (4): 199-204.

8. Brooten D, Roncolli M, Finkler S, Arnold L, Cohen A, Mennuti M. A randomized trial of early hospital discharge and home follow-up of women having caesarean birth. Obstet Gynecol. 1994; 84 (5): 832-9.

9. Smith-Hanrahan C, Deblois D. Pospartum early discharge: impact of maternal fatigue and functional ability. Clin Nurs Res 1995; 4 (1): 50-66.

10. Gagnon A, Edgar L, Kramer M, Papageorgiou A, Waghorn K, Klein M. A randomized trial of a program of early postpartum discharge with nurse visitation. Am J Obstet Gynecol 1997; 176 (1): 205-11.

11. Winterburn S, Fraser R. Does the duration of postnatal stay influence breast-feeding rates at one month in women giving birth for the first time? A randomized control trial. J Adv Nurs 2000; 32 (5): 1152-7.

12. Yanover MJ, Jones D, Miller MD. Perinatal care of low-risk mothers and infants. Early discharge with home care. N Engl J Med 1976; 294 (13): 702-5.

13. Ducel G, Fabry J, Nicolle L. Prevención de las infecciones nosocomiales. Guía práctica. Segunda edición Malta: Organización Mundial de la Salud; 2003.

14. Observatorio de la Calidad de la Atención en Salud (indicadores de calidad). Ministerio de la Protección Social, República de Colombia [Internet] [acceso: enero de 2011]. Disponible en: http://201.234.78.38/ocs/ public/ind calidad/indicador.aspx?indicador $\mathrm{id}=25$.

15. Hauck K, Zhao Z. ¿How dangerous is a day in hospital? A model of adverse events and length of stay for medical impatients. Med. Care. 2011; (12): 1068-75.

16. Ramírez-Villalobos D, Hernández-Garduño A, Salinas A, González D, Walker D, Rojo-Herrera G et ál. Early hospital discharge and early puerperal complications. Salud Publica Mex. 2009; 51: 212-8.

17. Ford J, Algert C, Morris J, Roberts C. Decreasing length of maternal hospital stay is not associated with increased readmission rates. Aust. N. Z. J. Public Health. 2012; 36 (5): 430-4.

18. Petrou S, Boulvain M, Simon J, Maricot P, Borst F, Perneger T et ál. Home-based care after a shortened hospital stay versus hospital-based care postpartum: an economic evaluation. BJOG. 2004; 111(8):800-6.

19. Sainz-Bueno J, Romano M, Teruel R, Benjumea A, Palacín A, González C et ál. Early discharge from obstetrics-pediatrics at the Hospital of Valme, with domiciliary follow up. Am. J. Obstet. Gynecol. 2005; 193 (3 pt 1): 714-26. 